\title{
The Jefferson Lab 12 GeV Upgrade
}

\author{
R. D. McKeown \\ Thomas Jefferson National Accelerator Facility, Newport News, Virginia 23606, USA \\ Department of Physics, College of William and Mary, Williamsburg, Virginia 23187, USA \\ E-mail: bmck@jlab.org
}

\begin{abstract}
Construction of the $12 \mathrm{GeV}$ upgrade to the Continuous Electron Beam Accelerator Facility (CEBAF) at the Thomas Jefferson National Accelerator Facility is presently underway. This upgrade includes doubling the energy of the electron beam to $12 \mathrm{GeV}$, the addition of a new fourth experimental hall, and the construction of upgraded detector hardware. An overview of this upgrade project is presented, along with highlights of the anticipated experimental program.
\end{abstract}

\section{Introduction}

Since 1995, the CEBAF facility at Jefferson Laboratory has operated high-duty factor (continuous) beams of electrons incident on three experimental halls, each with a unique set of experimental equipment. As a result of advances in the performance of novel superconducting radiofrequency (SRF) accelerator technology, the electron beam has exceeded the original $4 \mathrm{GeV}$ energy specification, and beams with energies up to $6 \mathrm{GeV}$ with currents up to $100 \mu \mathrm{A}$ have been delivered for the experimental program. In addition, the development of advanced GaAs photoemission sources has enabled high quality polarized beam with polarizations up to $85 \%$. The facility serves an international scientific user community of over 1200 scientists, and to date over 160 experiments have been completed.

Shortly after the first experiments were started, planning began to upgrade the capability of this facility to enable beams up to $12 \mathrm{GeV}$ in energy. In 2002, the Long Range Plan of the US Nuclear Science Advisory Committee (NSAC) [1] contained a recommendation: "We strongly recommend the construction of CEBAF at Jefferson Laboratory to $12 \mathrm{GeV}$ as quickly as possible". In March 2004 the US Department of Energy (DOE) granted CD-0 approval to develop a conceptual design for such a facility. In 2007, the NSAC Long Range Plan [2] reaffirmed the community commitment to this project: "We recommend completion of the 12 GeV Upgrade at Jefferson Lab. The Upgrade will enable new insights into the structure of the nucleon, the transition between the hadronic and quark/gluon descriptions of nuclei, and the nature of confinement." Considerable effort by the Jefferson Lab user community and the Laboratory staff facilitated DOE final approval for the start of construction in September 2008.

\section{Description of Construction Project}

Currently, CEBAF is a recirculating linac, with 2 linac sections consisting of 20 cryomodules each, each cryomodule containing 8 superconducting RF cavities. These cryomodules are each capable of an average $25 \mathrm{MeV}$ of acceleration, nominally producing $0.5 \mathrm{GeV}$ acceleration in each linac section. The recirculating arcs contain quadrupole and dipole magnets in separate 


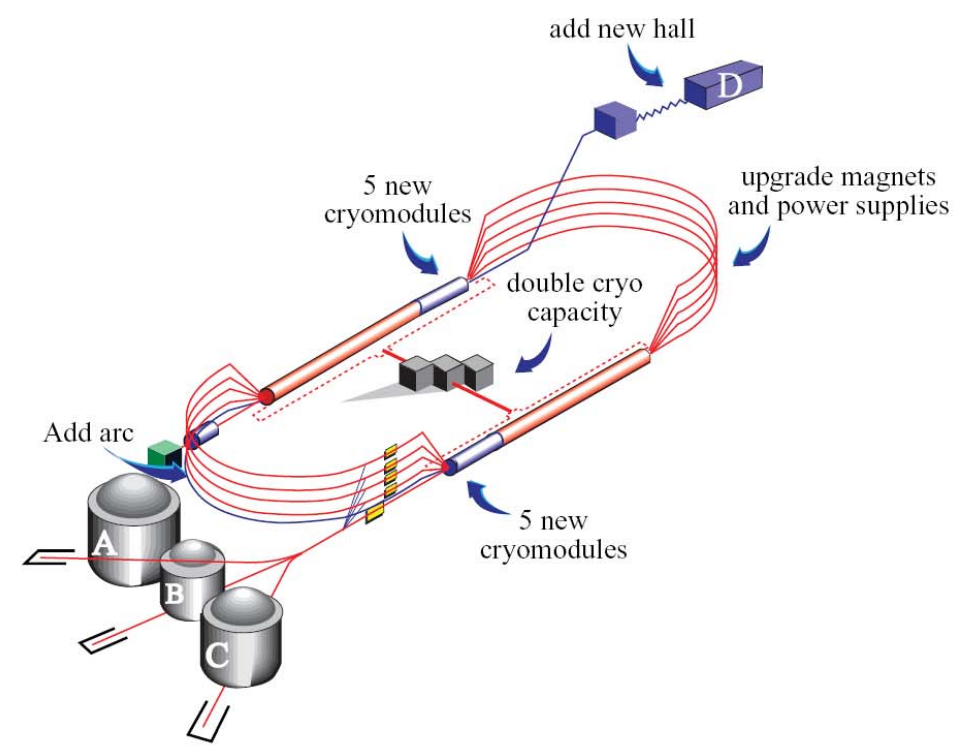

Figure 1. Jefferson Lab $12 \mathrm{GeV}$ upgrade concept.

beamlines that enable the beam to be accelerated up to 5 times through both linacs, producing a nominal energy of $5 \mathrm{GeV}$ with actual performance up to $6 \mathrm{GeV}$. After the second (south) linac, beam pulses can be "kicked" into an extraction line into one of the 3 experimental halls. Thus the $1497 \mathrm{MHz}$ microstructure can be split into three $499 \mathrm{MHz}$ beams with energies in multiples of $\frac{1}{5}$ of the full 5 -pass energy.

The basic concept of the $12 \mathrm{GeV}$ upgrade project is illustrated in Fig. 1. In addition to the upgrade of the accelerator system to enable delivery of $12 \mathrm{GeV}$ beam, the experimental equipment will be enhanced to facilitate full exploitation of the higher energy beam. This includes substantial new equipment in Hall B and Hall C, and a completely new Hall D with a new detector spectrometer system. The plan for Hall A also includes upgraded and new equipment that is outside the present construction project.

\subsection{Accelerator system upgrade}

The heart of the accelerator upgrade design is the addition of 5 additional cryomodules in each linac section. The original CEBAF construction project left extra drift space at the end of each linac section, enabling an energy upgrade. The new cryomodules will contain 7 -cell cavities instead of the 5-cell cavities in the original CEBAF. In addition, improved surface treatments now enable higher RF fields. The result is that each cryomodule will be capable of $100 \mathrm{MeV}$ of acceleration (compared to $20 \mathrm{MeV}$ in the original design specification). Thus each linac will now be capable of $1.1 \mathrm{GeV}$, and each recirculated beam will be able to reach $2.2 \mathrm{GeV}$. With upgrades to the arc magnets and power supplies, we would be able to deliver beams to all 3 existing experimental halls at energies up to $11 \mathrm{GeV}$, with beam available in multiples of $\frac{1}{5}$ of the full 5-pass energy. For example at full energy, beam would be available at three of the values corresponding to $2.2 \mathrm{GeV}, 4.4 \mathrm{GeV}, 6.6 \mathrm{GeV}, 8.8 \mathrm{GeV}$, and $11 \mathrm{GeV}$. The intensity of these beams may total up to $85 \mu \mathrm{A}$ with high polarization (up to $85 \%$ ) available.

A fifth arc of magnets will be added to the 2 nd recirculating arc, so that one beam can be accelerated through the north linac one more time (to reach a total of $12 \mathrm{GeV}$ before transmission to the new experimental Hall D. In addition, the central helium liquifier capacity is being doubled 
to provide increased delivery of liquid helium.

\subsection{Hall A upgrade}

Hall A will retain the present capability with 2 High Resolution Spectrometers (HRS), and will host new experimental equipment to be funded outside the present scope of the $12 \mathrm{GeV}$ upgrade project. As an example, the proposed Super Big-Bite Spectrometer (SBS) will be sited in Hall A. This is a larger version of the recently deployed Big-Bite Spectrometer. The SBS will consist of a large 48D48 dipole magnet followed by large area tracking (GEM detectors), time-of-flight scintillators, and particle ID detectors (Cerenkov and calorimeter). The solid angle acceptance depends on the deployment geometry but is typically $50-100 \mathrm{msr}$, and the spectrometer will be capable of operation at high luminosities exceeding $10^{38} / \mathrm{cm}^{2}$-s.

Hall A will also be the site of future high-precision parity violation experiments to provide stringent tests of the standard electroweak model. Measurements of parity-violating deep inelastic scattering will be pursued with the proposed solenoidal detector SOLID in Hall A.

\subsection{Hall B upgrade}

Hall B was the home of the CEBAF Large Acceptance Spectrometer (CLAS). This apparatus will be replaced by a new spectrometer, CLAS12, shown in Fig. 2, CLAS12 is designed to enable higher luminosity operation with electron beams, up to $10^{35} / \mathrm{cm}^{2}$-s, and improved particle identification at forward angles. The magnetic system includes a $5 \mathrm{~T}$ superconducting solenoid for central tracking, and a superconducting toroid at forward angles. The high field solenoid also shields the detector system from the high flux of Møller electrons generated in the target.

The forward detector system includes a high threshold cerenkov counter (HTCC) with pion threshold of $4.9 \mathrm{GeV}$ mounted in front of the toroid. The toroid and forward drift chamber system are designed to track charged particles in the range $5-40^{\circ}$, and are followed by a low threshold cerenkov counter (LTCC). The existing electromagnetic calorimeter (EC) is being upgraded with a pre-shower counter. The central detector system includes a silicon vertex tracker and a scintillator time-of-flight array.

\subsection{Hall C upgrade}

In Hall C, the existing high momentum spectrometer (HMS) will be complemented with a new super high momentum spectrometer (SHMS). The SHMS design is a QQQD with a horizontal bend, capable of a maximum central momentum of $11 \mathrm{GeV}$ with -11 to $+22 \%$ momentum acceptance. The solid angle acceptance is $5 \mathrm{msr}$ and the momentum resolution will be better than $10^{-3}$. The SHMS detector package includes a drift chamber system for tracking, quartz and scintillator hodoscopes, a noble gas Cerenkov counter and a heavy gas Cerenkov counter, and a lead glass calorimeter.

\subsection{Hall D}

The new experimental Hall D will be the host of the GlueX experiment (see Fig. 3). As discussed below, the main goal of the Gluex experiment is to search for exotic mesons produced in photoproduction on the nucleon. Hall D will be preceded by a photon tagging system. Linearly polarized photons will be produced by coherent bremmstrahlung on a thin $(\sim 20$ micron) diamond wafer. The scattered electrons from 8.5-9 GeV photons will be tagged with scintillator detectors following a bending magnet, yielding a tagged photon resolution of $0.2 \%$ with expected fluxes to reach $10^{8} / \mathrm{s}$.

The Gluex detector system includes a superconducting solenoid with $2.2 \mathrm{~T}$ field over a length of $495 \mathrm{~cm}$ and with an inner bore of $185 \mathrm{~cm}$. The $30 \mathrm{~cm}$-long liq- uid hydrogen target is surrounded by scintillation counters (START), a cylindrical drift chamber array (CDC) and an 


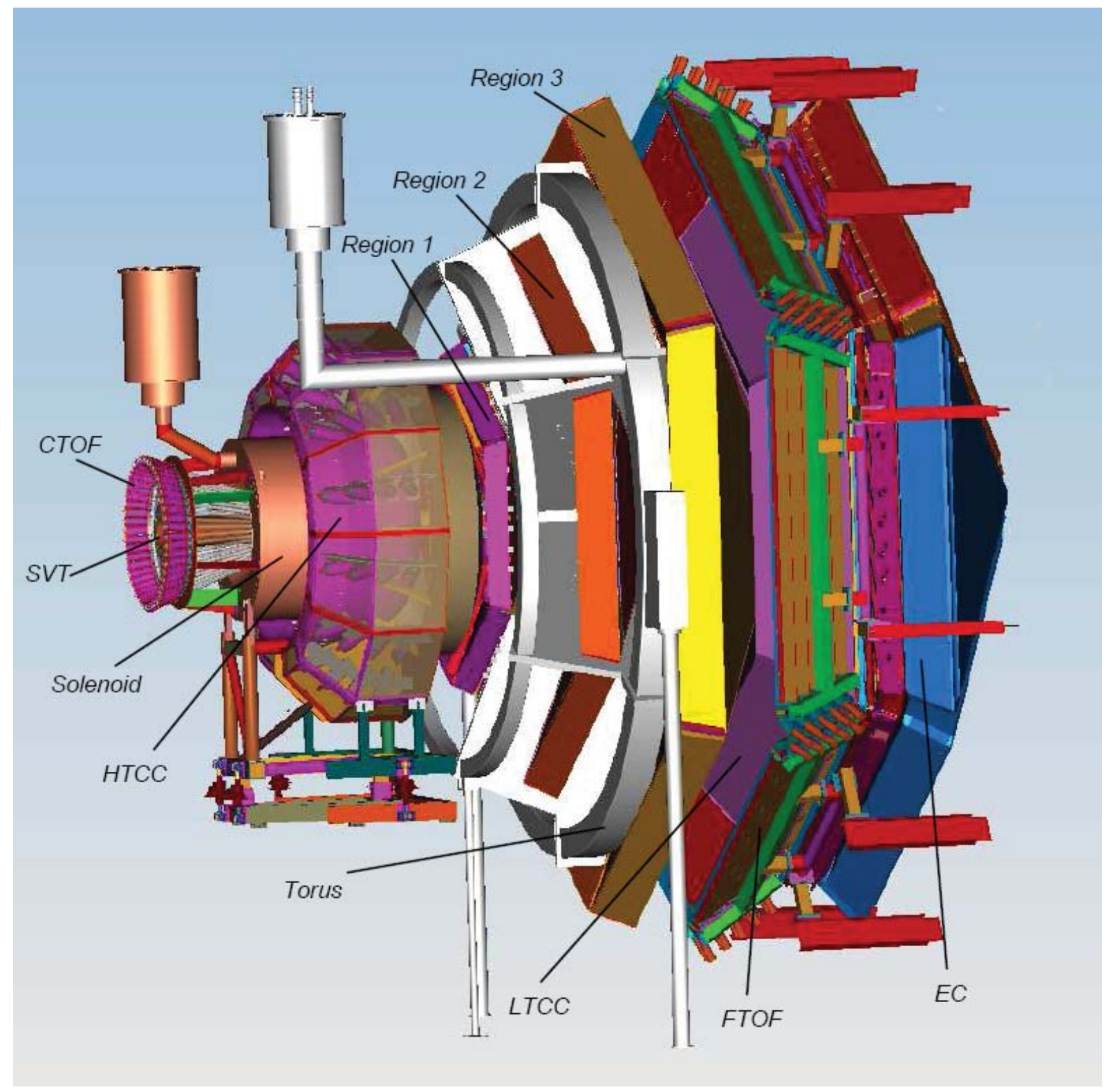

Figure 2. 3D view of CLAS12. The beam is incident from the left. The target is located inside the solenoid magnet.

electromagnetic lead/scintillating fiber calorimeter with a barrel geometry (BCAL). Downstream of the target are an array of planar drift chambers (FDC). Outside and downstream of the clear bore of the magnet are a Cerenkov counter, a wall of scintillation counters (TOF) to measure time-of-flight and an electromagnetic calorimeter (FCAL) consisting of lead-glass blocks. The angular coverage will be from $1-120^{\circ}$ for charged particles, and from $2-120^{\circ}$ for photons.

\section{Physics Program}

The physics program to be addressed with the Jefferson Lab $12 \mathrm{GeV}$ upgrade has been developed in collaboration with the user community and with the guidance of the Program Advisory Committee. There are presently 34 approved experiments, and 13 additional proposals have 


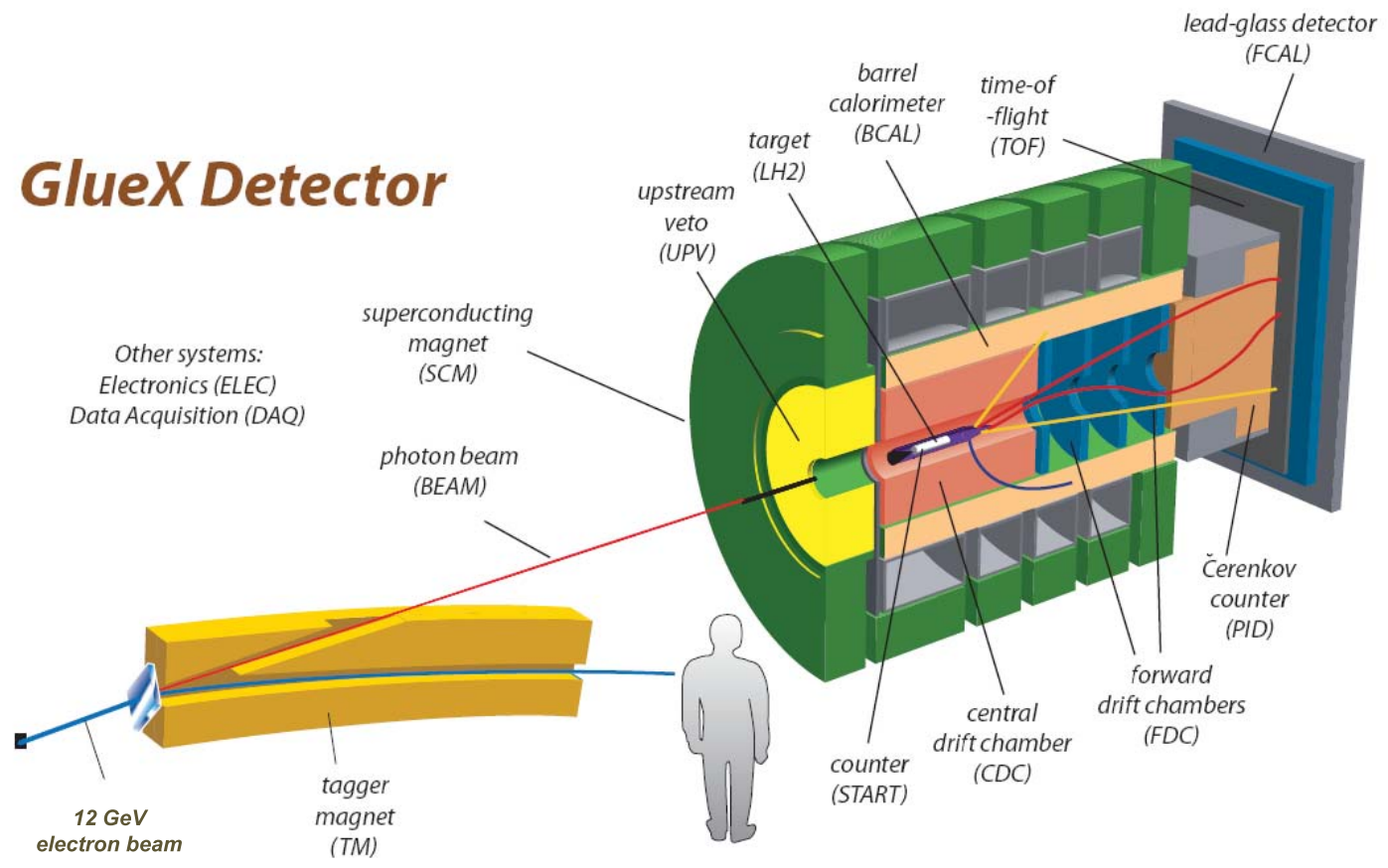

Figure 3. Schematic of GlueX apparatus.

conditional approval. These proposals fall into four general categories:

- the physical origins of quark confinement (including meson and baryon spectroscopy),

- the spin and flavor structure of the proton and neutron,

- the quark structure of nuclei,

- discovery of new physics through high precision tests of the Standard Model.

It is not possible here to provide a comprehensive review of this broad program, so a few selected highlights will be presented.

\subsection{Meson Spectroscopy with GlueX}

The GlueX experiment [3] will provide unprecedented capability to study the spectrum of light quark mesons in photoproduction. A major goal is to search for mesons with exotic quantum numbers $\left(J^{P C}=0^{+-}, 1^{-+}, 2^{+-}\right)$that cannot be described by quark-antiquark states. One can construct such states in models [4] that incorporate degrees of freedom associated with excitation of a gluonic flux tube connecting the quark and antiquark. More recently, these states and their properties have been studied in detail using lattice QCD methods [5], and the lattice calculations support the interpretation of excitation of gluonic degrees of freedom.

\subsection{Nucleon Spin Structure}

The study of the spin structure of the nucleon is still a very active area of research. For the last 25 years, studies of the spinm -dependent parton distributions have indicated that the quark helicities contribute a small fraction of the nucleon spin [6]. Recent data from RHIC and semi-inclusive Deep Inelastic Scattering (SIDIS) indicate that the gluon spin contribution 
is also small [7]. So although there has been considerable progress in constraining the spindependent parton distribution functions, a satisfactory accounting of the angular momentum of the nucleon in terms of its fundamental constituents is still lacking. It now appears that the orbital angular momentum of the quarks may play a substantial role, and new experimental efforts are being proposed to address this issue. There has been a great deal of theoretical progress in the development of experimental observables that could reveal aspects of the quark orbital angular momentum. These include generalized parton distributions (GPD) that can be accessed through deeply virtual Compton scattering (DVCS) [8, 9], and transverse momentum distributions [10] which can be studied in semi-inclusive deep inelastic scattering (SIDIS). The large acceptance available with the CLAS12 detector system makes it well-suited to the study of DVCS [11. Measurements of TMD with SIDIS will be an important topic for the proposed SOLID spectrometer in Hall A.

\subsection{Testing the Standard Model}

The discoveries of dark matter, dark energy, and the flavor oscillations of neutrinos (associated with their small but finite masses) are all indications that the standard model of the strong and electroweak interactions requires modification. In addition, there are theoretical motivations for extending the standard model associated with protecting the Higgs mass from uncontrolled loop corrections. These issues generally lead to the view that the standard model is part of a larger theoretical framework, and such an extension of the theory should lead to observational consequences. Thus there are experimental activities associated with two general methods for addressing this issue. One is to advance the high energy frontier with higher energy particle accelerators to attempt to obtain evidence for new particles that can be produced at higher energy. The other method is to perform high precision measurements at lower energies to provide information that can indicate the properties of a more complete theory. Indeed, such precision measurements yield complementary information to that which can be obtained at the energy frontier. For a review of this approach, including discussion of parity-violating electron scattering, see [12].

The $12 \mathrm{GeV}$ upgrade of CEBAF will offer new opportunities for precision measurements, particularly in studies of parity violating electron scattering. The neutral weak interaction violates parity symmetry, and so measurement of parity violation in electron scattering is a consequence of interference of neutral weak and electromagnetic amplitudes. Extensions of the standard model may involve the existence of new massive $\left(M>M_{Z}\right)$ neutral bosons that couple to electrons and/or quarks. Such phenomena, or other similar processes, would lead to changes in the parity-violating interaction with electrons and thus manifest themselves in parity violation experiments.

There are presently 2 proposals to perform such measurements at the upgraded CEBAF. One would use the SOLID solenoidal magnetic spectrometer system to study parity-violating deep inelastic scattering [13]. The other proposal involves the construction of a novel dedicated toroidal spectrometer to study parity-violating Møller scattering [14]. Both experiments propose to be sited in experimental Hall A.

\section{Summary}

The $12 \mathrm{GeV}$ upgrade project at Jefferson Lab will enable a powerful new experimental program that will advance our understanding of the quark/gluon structure of hadronic matter, the nature of Quantum Chromodynamics, and the properties of a new extended standard model of particle interactions. Commissioning of the upgraded beam will be begin in 2013, and the full complement of upgraded experimental equipment will be completed in 2015. This unique facility will provide many opportunities for exploration and discovery for a large international community of nuclear scientists. 


\section{Acknowledgements}

I am grateful to Allison Lung, Kees de Jager, Volker Burkert, Steve Wood, and Eugene Chudakov for their assistance in preparing this presentation. This work was supported by DOE contract DE-AC05-06OR23177, under which Jefferson Science Associates, LLC, operates the Thomas Jefferson National Accelerator Facility.

\section{References}

[1] 2002 Long Range Plan, Nuclear Science Advisory Committee, http://dnp.nscl.msu.edu/nplinks/lrps/2002_lrp.pdf.

[2] 2007 Long Range Plan, Nuclear Science Advisory Committee, http://dnp.nscl.msu.edu/nplinks/lrps/2007-Nuclear-Science.High-Res.pdf .

[3] Jefferson Lab proposal E12-06-102.

[4] N. Isgur and J. Paton, Phys. Rev. D31, 2910 (1985).

[5] J. J. Dudek, et al., Phys.Rev.D82 034508 (2010).

[6] S. D. Bass, Rev. Mod. Phys.77, 1257-1302 (2005).

[7] D. de Florian, et al., Phys. Rev. Lett. 101 072001, (2008).

[8] X. Ji, Phys. Rev. Lett. 78, 610 (1997); Phys. Rev. D55, 7114 (1997).

[9] A.V. Radyushkin, Phys. Rev. D56, 5524 (1997).

[10] J. C. Collins, Acta Phys. Pol. B34, 3103 (2003), and references therein.

[11] Jefferson Lab proposal E12-06-119.

[12] M. J. Ramsey-Musolf and S. Su, Phys. Rept. 4561 (2008).

[13] Jefferson Lab proposal E12-10-007.

[14] Jefferson Lab proposal E12-09-005. 